\title{
Lipedema: a clinical entity
}

\author{
Paweł Łyszczak, Andrzej Szuba \\ Department of Internal Diseases, $4^{\text {th }}$ Military Clinical Hospital in Wroclaw, Poland \\ Division of Angiology, Faculty of Health Sciences, Wroclaw Medical University, Poland
}

\begin{abstract}
Lipedema is a chronic, progressive disorder of adipose tissue, found almost exclusively in women, that involves disproportionate subcutaneous fat depositions, leading to progressive and symmetric enlargement of the legs. The condition usually appears at puberty or in the third decade of life. Many patients report a family history of such disorder. The diagnosis is usually based on the physical examination and medical history. If left untreated, lipedema may result in secondary lymphatic dysfunction, physical impairment and mental problems, leading to significant damage to patient's quality of life. The condition is frequently misdiagnosed as lymphedema or mistreated as obesity. Other than that, the differential diagnosis includes chronic venous insufficiency, constitutional variability of the legs, lipohypertrophy, Dercum's disease, Madelung's disease. The treatment options include diet and compression therapy, conservative treatment of secondary edema and surgical interventions. There is an urgent need for further studies on the pathogenesis of lipedema, epidemiology, diagnostic criteria and possible curative treatment.
\end{abstract}

Key words: lipedema, lymphedema, obesity, edema

Acta Angiol 2018; 24, 4: 14I-148

\section{Introduction}

Lipedema was first described in 1940 by Allen and Hines as bilateral enlargement of the legs due to abnormal depositions of subcutaneous fat associated with often mild edema [I, 2]. Lipedema, as well as multiple symmetric lipomatosis (MSL) and Dercum's disease (DD), has been classified to rare adipose disorders (RADs) [3]. Even though the condition has not yet been registered in the International Classification of Diseases (ICD-I0) of the World Health Organization, the current knowledge indicates that lipedema is a clinical entity, distinct from lymphedema. Patients usually develop bilateral, symmetrical, non-pitting enlargement of lower extremities, disproportionately greater than that of the trunk and upper extremities. In a typical presentation, the feet are unaffected and the Stemmer sign is absent. Patients often report pain of affected areas, tenderness and spontaneous or minimal trauma-induced bruising [4]. The course of lipedema is variable. Patients may develop serious complications, which include lipolymphedema, orthopedic disorders, immobility-related obesity and major mental distress. Therefore, it is crucial that patients suffering from lipedema receive an accurate diagnosis as early as possible, followed by optimal information and treatment. In this article, the epidemiology, pathogenesis, clinical presentation, diagnostic findings, differential diagnosis and treatment are presented. The aim of this review is to increase the awareness and knowledge of lipedema among physicians resulting in optimizing management and care of patients with this condition.

\section{Epidemiology}

No epidemiological data on the prevalence of lipedema is yet available. The studies from speciality lymphedema clinics estimate that 10-18\% of referred

Address for correspondence: Paweł Łyszczak, Department of Internal Diseases, $4^{\text {th }}$ Military Clinical Hospital in Wroclaw, R. Weigla 5, 50-98। Wroclaw, Poland, e-mail: pa.lyszczak@gmail.com 
patients were eventually diagnosed with lipedema [5-7]. Other authors estimate that up to $11 \%$ of females may be affected [8]. Surely, there is a significant underestimate due to the failure of referral by physicians and common misdiagnosis.

Although a few cases of men with lipedema $[2,9,10]$ have been reported in the literature, the condition affects almost exclusively females.

Significant differences in prevalence in different parts of the world are noted, e.g. the condition is practically absent among women of Asian origin [ I I]. The most data concerning management of lipedema is obtained from Germany, where the diagnosis and treatment of this condition are well developed.

\section{Pathogenesis and etiology}

The etiology of lipedema remains uncertain. Several theories concerning this condition have been presented.

Földi and Földi [12], based on their histological findings, suggested that microangiopathy in the area of the affected adipose tissue results in higher permeability to proteins and increased capillary fragility. In the further course of this condition, impairment of lymph transport capacity by overgrown adipose tissue and excess of extravasated fluid induce fibrosis and lead to subsequent progression to lipolymphedema [13].

Amman-Vesti et al. described lymphatic microaneurysms in lipedema patients suggesting the involvement of lymphatic system in lipedema pathogenesis [14].

Lymphatic system failure might contribute to fat deposition. Mice with heterozygous Prox-I mutation present with lymphatic system failure and obesity [ I5]. Lymph itself was found to be a potent stimulator of adipogenesis [16].

Vascular endothelial growth factor (VEGF) is considered to be a potent inducer of angiogenesis. Siems etal. [17] discovered fourfold higher levels of plasma VEGF in lipedema patients. This may result in pathological angiogenesis and increased capillary fragility. Further studies reported high levels of both protein carbonyls and malonyldialdehyde (MDA), which are known indicators of oxidative stress, representing an accelerated lipid peroxidation in lipoedematous tissue.

In other study, Suga et al. [18] analyzed differences between lipedema tissue and the nonaffected adipose tissue obtained from a patient with severe lipedema. Immunohistochemical findings proved edematous change, the presence of large adipocytes and crown-like structures (necrotizing adipocytes surrounded by infiltrating CD68+ macrophages) in lipedema tissue. Furthermore, the authors discovered proliferation of $\mathrm{Ki} 67+$ and CD34+ cells, which are identified as adipose-derived stem cells. The hypothesis has been presented that the increased proliferation of adipose stem cells leads to a rapid increase in adipogenesis, resulting in localized hypoxia, adipocyte necrosis and macrophage recruitment.

As a positive family history of lipedema is one of its characteristics, genetic inheritance has been studied. Child et al. [19] proposed, that lipedema is a genetic disorder with either $\mathrm{X}$-linked dominant inheritance or autosomal dominant inheritance with sex limitation. Bano et al. [20] reported a case study that identifies a Pit-I mutation in members of a family with lipedema.

The condition has not been yet included in the Online Mendelian Inheritance in Man (OMIM) database for hereditary diseases.

To fully understand the pathophysiological mechanisms of lipedema further studies are urgently needed.

\section{Clinical presentation}

There are only few published criteria for lipedema diagnosis. On that basis, the diagnosis of lipedema depends mainly on the clinical experience of the medical professionals. Physical examination should include thorough evaluation of extremities affected with the edema. Especially asymmetry or any other differences between the limbs should be noted. It is important to meticulously access the location, volume and nature of limb enlargement. Prior surgical interventions in the extremities and groin area should be documented [2I]. Patients typically present bilateral, symmetrical increase of adipose tissue on the lower limbs. The condition affects buttocks, as well as hips, thighs and calves [10]. In the initial phase, the first sign could be the filling of the retromalleolar sulcus, that is the disappearance of the concave spaces on both sides of the Achilles tendon [22]. Characteristically, the feet are spared [23] with negative Stemmer sign. The fatty enlargement of the extremity begins abruptly above the malleolus. The visible demarcation between the normal and abnormal tissue near the ankle-distinct step-off — is accentuated, which is known as "cuff sign" [4]. That is an important morphologic criterion of lipedema. In some patients, similar deposits develop in the upper limbs, with sparing of the hands. Although the majority of patients suffering from lipedema are overweight or obese, there are also some of normal weights. What differs lipedema from obesity is the disproportionate enlargement of the lower extremities in relation to the trunk and upper extremities. The weight loss has usually only limited influence on the disproportionate fat distribution. The fatty swelling is typically not reversible overnight or upon elevation of the extremity. The fatty tissue is tender under pressure and likely to develop hematomas even after the slightest trauma. Patients often report the sensations of heaviness and discomfort of the legs. 
There are five types of lipedema based on the area of accumulation of excessive subcutaneous adipose tissue: Type I - buttocks and thighs.

Type II - extends to knees with the formation of fat folds on the inner side of the knees.

Type III - buttocks to ankles.

Type IV - involving the legs and arms.

Type V - lipo-lymphedema [24].

Meier-Vollrath and Schmeller [25] proposed a staging system to assess the severity of lipedema. In stage I the skin surface is normal with enlarged subcutis, on palpation, multiple small nodules ('styrofoam balls') can be distinguished. Stage II is associated with the development of bigger nodules ('walnut- to apple-like indurations'). As a result, the overlying skin appears doughy, puckering and uneven ("mattress phenomenon"). In stage III larger indurations and deforming fat deposits may develop and orange-peel appearance of the skin may be visible.

Lipedema onset is typical during teenage years by the third decade of life but can occur at other times of hormonal change such as pregnancy or even menopause [19].

Large variability in the course of the condition is noted. Some patients develop only mild lipedema, which does not progress. Others show gradual and slow progression. Sudden exacerbations may be caused by the stressful situation, such as pregnancy or surgery [4]. Obesity is perceived as a major exacerbating factor in the course of lipedema. At any time the condition may be complicated by secondary lymphedema [26]. Furthermore, long-lasting lipedema linked with morbid obesity may lead to immobility-induced edema. With the progression of lipedema, the increased lower extremities weight may lead to joint damage causing orthopedic disorders (e.g. genu valgum, pes planus) and gait alternations with impaired mobility [7].

\section{Diagnostic findings}

There are no blood or urine biomarkers for lipedema. Although it is usually possible to diagnose lipedema based only on the clinical features and medical history, patients often undergo advanced radiologic and radionuclide imaging. Data provided from these investigations might be used for differential diagnosis and evaluation of the severity of lipedema.

High-resolution cutaneous ultrasonography can be used to differentiate lipedema from lymphedema. Patients with lipedema have normal dermal thickness and echogenicity, whereas increased dermal thickness and decreased echogenicity are found in lymphedema patients [27]. Duplex examination (Doppler colour ultrasound) of lipedema reveals thickened subcutis with high echogenicity, while in lymphedema echodense, non-compressible areas are observed [7].

Computed tomography images provide high sensitivity and specificity of $100 \%$ for the diagnosis of lipedema. Typical scans show diffuse and homogenous lipomatous hypertrophy of subcutaneous tissue without skin abnormalities. Images of skin thickening, subcutaneous fluid accumulation, honeycomb appearance and muscle enlargement are characteristic of lymphedema [28].

Magnetic resonance imaging of lipedema shows normal skin thickness and lipomatous hypertrophy, while skin thickening, subcutaneous tissue thickening and honeycomb appearance are associated with lymphedema [29, 30]. Magnetic resonance lymphangiography is considered useful when evaluating lymphatic insufficiency in lipedema/lipolymphedema patients [3I].

Lymphoscintigraphy in lipedema patients shows normal or initially even increased lymphatic function in contrast to lymphedema [32, 33]. In long-lasting lipedema lymphoscintigraphy findings prove lymphatic insufficiency, often without morphologic abnormalities observed in lymphedema [34].

Amann-Vestii et al. [14] using fluorescence microlymphography with fluorescein isothiocyanate (FITC) proved that multiple microplymphatic aneurysms of lymphatic capillaries in the diseased skin region of lipedema might be distinguishing finding in patients with lipedema. In lipedema lymphatic drainage visualized in the microlymphography is typically intact or only unremarkably impaired, in contrast with lymphedema, where the drainage is significantly impeded [35].

Near-infrared fluorescence (NIRF) lymphatic imaging with indocyanine green (ICG) might be the next useful tool in accessing the lymphatic function in patients with edema of uncertain origin. NIRF lymphatic imaging phenotypes have already been established for patients with lymphedema and Dercum's disease [36].

\section{Differential diagnosis}

The most important differential diagnoses of lipedema include lymphedema and obesity. In most cases, the thorough physical examination and extensive anamnesis are enough to establish the right diagnosis.

Lymphedema refers to localized swelling, typically of one extremity, secondary to lymphatic dysfunction. Patients often report a history of erysipelas, cellulitis or prior malignancy followed by its treatment (surgery or radiation therapy). Secondary lymphedema of the lower extremities is estimated to occur in up to $25 \%$ of patients who underwent surgery and radiotherapy for the treatment of gynecological cancer [37]. Filariasis is also associated with secondary lymphedema in areas where the disease is endemic. Contrary to lipedema, 
the swelling in lymphedema is typically unilateral and non-painful. Unlike in lymphedema [38, 39], the Stemmer's sign (inability to pinch the base of the second toe) is negative in lipedema [40]. Furthermore, lymphedema, as opposed to lipedema, responds well to compression therapy [4I]. The skin in patients suffering from lymphedema is often discoloured, warty and sclerotic, whereas the skin in lipedema remains intact.

Often, in long-lasting patients, lipedema may be complicated by lymphedema or obesity. Therefore, it is crucial to know the patient's historical symptoms and the course of the disease.

Distinguishing obesity from lipedema might come as challenging. What can be misleading, many patients with lipedema are seen with an apparently overweight BMI > 25 [19]. However, there are few diagnostic criteria, which denote obesity. Excess fatty tissue in obese patients appears in all parts of the body, unlike lipedema, where feet and hands are spared. In obese patients, the adipose tissue is distributed in a generalized pattern, whereas in lipedema the disproportion of fat accumulation between upper and lower body is noticeable. BMI might not be useful since it takes total weight into consideration but not the disproportionate distribution of the fatty tissue. However, the waist-hip ratio or waist-height ratio in obesity are abnormally elevated, unlike in lipedema. Obesity responds to restricted dietary intake and exercising. On the contrary, lipedema patients often report aggressive dietary plans that fail to result in weight loss in the waist and lower extremities $[10,19]$. Furthermore, the subcutaneous fatty tissue in obesity is not pain-sensitive. A more comprehensive list of differences between lipedema, lymphedema and obesity is presented in Table I.

Other, less common conditions should be considered in differential diagnosis as well.

Chronic venous insufficiency (phleboedema) causes hyperpigmentation, teleangiectasies, purpura, leg ulcers, pitting edema that decreases after bed rest and exercising - symptoms typically absent in lipedema patients. Edema responds well to compression therapy [42]. Varicose veins are often present in patients with lipedema, thus should not me considered as a distinguishing feature [2, 19].

Lipohypertrophy is a condition that is characterized by localized symmetrical fat depositions in buttocks, the upper or lower thighs or the lower calves [43]. Feet are spared. In contrast to lipedema, there is no pain on pressure, edema and malleolar fat pads are absent $[6,44]$. The tendency to lipohypertrophy of the legs and gluteal region without displaying further signs of lipedema is often treated as a constitutional variant of normal leg form [45] and associated with ethnicity (e.g. women from the Mediterranean region).

Madelung's disease (MSL, multiple symmetric lipomatosis) is a condition that involves tumour-like non-encapsulated fatty deposits in the neck, upper trunk, upper back and occasionally the limbs. Typically, Madelung's disease affects white, middle-aged men of Mediterranean origin who have a history of alcohol overuse. Diabetes mellitus, lipid disorders, hypothyroidism and liver disease are common comorbidities [46, 47].

Table I. Differential diagnosis of lipedema, lymphedema and obesity

\begin{tabular}{|c|c|c|c|}
\hline & Lipedema & Lymphedema & Obesity \\
\hline Gender & Females almost exclusively & Males and females & Males and females \\
\hline Family history & Frequent & In primary lymphedema & Frequent \\
\hline Onset & $10-30$ years old (typically at puberty) & $\begin{array}{l}\text { Childhood (primary) } \\
\text { Any age (secondary) }\end{array}$ & Childhood onward \\
\hline Area affected & $\begin{array}{l}\text { Bilateral and symmetric involvement } \\
\text { of legs, thighs, buttocks (feet spared); } \\
\text { arms }\end{array}$ & $\begin{array}{l}\text { In primary lymphedema lower } \\
\text { extremities more frequent, typical } \\
\text { asymmetric involvement }\end{array}$ & $\begin{array}{l}\text { All parts of the body, } \\
\text { symmetrical }\end{array}$ \\
\hline Bruising & Yes & No & No \\
\hline Pain & Yes (tenderness or spontaneous pain) & No & No \\
\hline Skin & Normal consistency & $\begin{array}{l}\text { Thick, firm, sometimes reddish, } \\
\text { brownish, warty with hyperke- } \\
\text { ratosis }\end{array}$ & Normal consistency \\
\hline Stemmer's sign & Absent & Often present & Absent \\
\hline Pitting edema & Minimal & Usually present in variable severity & No \\
\hline Effect of dieting & Little effect on legs, arms and buttocks & Little effect on affected extremity & Symmetrical loss \\
\hline Effect of compression & Small & Positive & None \\
\hline Skin infections & Absent & Common & Absent \\
\hline
\end{tabular}


Dercum's disease (adiposis dolorosa) is a condition characterized by painful, easily bruised fatty tumours, which usually occurs in obese, post-menopausal women and is associated with type 2 diabetes, asthenia, weakness and mental phenomena such as depression, anxiety, confusion and dementia. Adipose depositions usually locate in the area of limbs, trunk, buttocks and pelvic area, with a possible progression to circumscribed or general diffuse type [48-5 I]. Familial occurrence with dominant inheritance has been also reported [52]. Hansson, et al. [53] proposed Dercum's disease classification including I. generalised diffuse form with widespread fatty tissue without clear lipomas, II. generalised nodular form with general pain in adipose tissue and intense pain in and around multiple lipomas, III. localised nodular form and IV. Juxta-articular form with painful lipomas associated with joints. The generalised diffuse form of Dercum's disease shares many similarities with lipedema [36]. Hence, lipedema and Dercum's disease are perceived by some experts as disorders in the same spectrum, if not the same condition in different stage of progression [3].

Acquired partial lipodystrophy (APL, Barraquer-Simons syndrome) leads to symmetrical and progressive loss of subcutaneous adipose tissue starting at the face and spreading to the upper part of the body, sparing the legs. Due to similar phenotype, the condition might be confused with lipedema [54]. APL etiology is associated with autoimmune response to viral infection [55]. In APL patients there is a visible loss of subcutaneous adipose tissue (SAT) from the upper body to the waist, in contrast to lipdema, that is associated with normal or increased volume of SAT in these areas.

Other conditions that cause edema of the lower extremities include idiopathic cyclic edema, edema due to internal disease (e.g. congestive heart failure, renal insufficiency), drug-induced edema and orthostatic edema. In contrast to lipedema, those usually involve pitting edema [4].

\section{Treatment}

As a causal treatment of lipedema is currently unavailable, the management of lipedema is symptom-based and aimed to reduce disability and patients' complaints as well as prevent progression. Treatment of lipedema should also target factors that negatively influence the course of the condition, such as obesity, lymph- and phleboedema, poor physical activity, incorrect insight into the disease $[I I, 56]$. Many patients will find the simple acknowledgement of their complaints relieving since they often have been lengthily misdiagnosed as obese or mistreated as lymphedema. Ineffectiveness of restrictive diets, exercising and unsatisfactory effects of lymphatic therapy result in patients' frustration, demotivation and disappointment.
There is a little accessible data concerning proven lipedema treatments and lifestyle behaviours that may be beneficial. Therefore, education and proper information are fundamental in the management of lipedema patients.

Lipedema is a chronic disease that is likely to cause pain, fatigue and deconditioning. That may result in deterioration in daily activity, shortage of physical exertion and increase in body weight [ $\mathrm{I}]$. Patients suffering from lipedema should be strongly and continuously encouraged to maintain physical activity, preferably aerobic exercises with low risk of injury such as speed walking, swimming and cycling [45]. Exercising activates muscle pump preventing lymphatic stasis, reducing edema formation and decreasing the risk of obesity. Dietician's support is also beneficial. Even though dietary changes will not reduce the disproportionate fat distribution, they may reduce the risk of general obesity, improve prognosis and general well being.

Lipedema may be detrimental to mental health. Women affected by lipedema are likely to report low self-esteem, lack of confidence, depression, eating disorders, self-harm tendencies or even suicidal thoughts. Furthermore, patients frequently state, that this condition has restricted their career, social and sex life [57]. Psychological support could be beneficial for some patients. Cognitive behaviour therapy is considered as a useful method in supporting the patient with this long-term condition in being proactive in managing their condition and helping patients with depression emotionally [58].

Treatment options include conservative approach and surgical interventions. Standard conservative therapy for lipedema can be performed with complex decongestive therapy, which is based on clinical experience. Its elements are compression bandaging, manual lymph drainage (MLD), intermittent compression therapy with multi-chamber devices and adequate skin care. Complex decongestive therapy does not affect fat tissue [59]. Therefore, therapeutic effects for patients with simple lipedema with unaltered lymphatic drainage can be unsatisfactory. Nevertheless, consistent manual lymph drainage combined with multilayer and multicomponent compression bandaging may lead to a reduction of pain and tendency to develop hematomas $[60,61]$. Furthermore, it has been proven to improve skin elasticity and restore veno-arteriolar reflex [62]. Conservative approach can highly contribute to the effective treatment of patients with lipolymphedema by preventing progression of the lymphatic component. Once the optimal effect of decongestive therapy is achieved, patients should be provided with flat knitted therapeutic compression hosiery to maintain the benefits of the therapy [63]. 
Surgical intervention remains the only available method to correct abnormal fat distribution. In contrast to the conservative treatment, it provides a reduction of mass. The most performed procedure is liposuction. In the past, this technique was perceived as contraindicated in lipedema patients due to the risk of iatrogenic damage to the lymphatic vessels [7]. The introduction of tumescent local anesthesia drastically reduced the risk of lymphatic impairment and significantly improved the outcomes of the liposuction [64, 65]. In tumescent liposuction, large amounts of fluids (saline, lidocaine, sodium bicarbonate and epinephrine) are infiltrated in the subcutaneous tissues and subsequently aspirated with vibrating microcannulas. The procedure leads to a distinct reduction of fat tissue. In addition to the positive cosmetic effect, it dramatically reduces symptoms such as bruising and pain. Furthermore, it has been proven, that it improves mobility. To maintain the reduced volume of the limb postoperatively patients should use compression garments [66] and keep a healthy lifestyle with balanced calorie intake and weight control. Although there are not well-recognized criteria of what is the optimal time in the course of lipedema to perform liposuction, latest studies showed that patients in stages II-III benefit the most from the procedure [65].

In some advanced cases of lipedema large localized deposits of fatty tissue are found. When situated on the legs it may result in severe mechanical limitations, leading even to inability to walk. In such cases, longitudinal excision (lumpectomy) is required [ $\mathrm{I}]$.

\section{Conclusion}

Current knowledge indicates that lipedema is an underdiagnosed clinical entity. The most common differential diagnoses include obesity, lymphedema and constitutional variants of normal leg form. Further studies on its pathogenesis, epidemiology and effective treatment are urgently needed. Hard objective criteria for lipedema diagnosis should be established. Actions to promote awareness of this condition among medical professionals should be taken to provide patients with accurate treatment.

\section{Conflict of interest}

None.

\section{References:}

I. Allen E, Hines EJ. Lipedema of the legs. A syndrome characterized by fat legs and orthostatic edema. Proc Staff Meet Mayo Clin. 1940; 15: 184-187.

2. WOLD LE, HINES EA, ALLEN EV. Lipedema of the legs; a syndrome characterized by fat legs and edema. Ann Intern Med. 1951; 34(5): 1243-1250, indexed in Pubmed: 14830102.
3. Herbst KL. Rare adipose disorders (RADs) masquerading as obesity. Acta Pharmacol Sin. 2012; 33(2): 155-172, doi: 10.1038/ aps.2011.153, indexed in Pubmed: 2230I856.

4. Langendoen SI, Habbema L, Nijsten TEC, et al. Lipoedema: from clinical presentation to therapy. A review of the literature. Br J Dermatol. 2009; 161(5): 980-986, doi: 10.1111/j.13652133.2009.094|3.x, indexed in Pubmed: 197856।0.

5. Gregl A. Das lipödem. Z Lymphol. 1987; II: 4I-3.

6. Herpetz U. Das lipödem. Z Lymphol. 1995; 19: I-II.

7. Cohen GM, Irby MB, Boles K, et al. Telemedicine and paediatric obesity treatment: review of the literature and lessons learnt. Clinical Obesity. 2012; 2(3-4): 103-1II, doi: 10.11। I/j.1758$8111.2012 .00050 . x$

8. Fonder MA, Loveless JW, Lazarus GS. Lipedema, a frequently unrecognized problem. J Am Acad Dermatol. 2007; 57(2 Suppl): SIS3, doi: 10.1016/j.jaad.2006.09.023, indexed in Pubmed: 17637360.

9. Chen SG, Hsu SD, Chen TM, et al. Painful fat syndrome in a male patient. Br J Plast Surg. 2004; 57(3): 282-286, doi: 10.1016/j.bjps.2003.12.020, indexed in Pubmed: 15006533.

10. Fife CE, Maus EA, Carter MJ. Lipedema: a frequently misdiagnosed and misunderstood fatty deposition syndrome. Adv Skin Wound Care. 2010; 23(2): 81-92; quiz 93, doi: 10.1097/01. ASW.0000363503.92360.9I, indexed in Pubmed: 20087075.

II. Halk $A B$, Damstra RJ. First Dutch guidelines on lipedema using the international classification of functioning, disability and health. Phlebology. 2017; 32(3): 152-159, doi: 10.1 177/02683555I663942 I, indexed in Pubmed: 27075680.

12. Földi E, Földi M. Strössenreuther R, Kubik S. Textbook of lymphology for physicians and lymphedema therapists. 2nd ed. URBAN \& FISCHER, Munchen 2006: 396-403.

13. Brauer WJ, Brauer VS. Age-dependent lymph transport in legs of patients with lipedema | Altersabhängigkeit des lymphtransportes beim lipödem und lipolymphödem. Lymphologie Forsch und Prax. 2005; 9(I).

14. Amann-Vesti BR, Franzeck UK, Bollinger A. Microlymphatic aneurysms in patients with lipedema. Lymphology . 2001; 34(4): 170-175.

15. Harvey NL, Srinivasan RS, Dillard ME, et al. Lymphatic vascular defects promoted by ProxI haploinsufficiency cause adult-onset obesity. Nat Genet. 2005; 37(10): 1072-1081, doi: 10.1038/ ng |642, indexed in Pubmed: 16170315.

16. Nougues J, Reyne Y, Dulor JP. Differentiation of rabbit adipocyte precursors in primary culture. Int J Obes. 1988; I2(4): 32I-333, indexed in Pubmed: 3198310.

17. Siems W, Grune T, Voss P, et al. Anti-fibrosclerotic effects of shock wave therapy in lipedema and cellulite. Biofactors. 2005; 24(I-4): 275-282, indexed in Pubmed: 16403988.

18. Suga $\mathrm{H}$, Araki J, Aoi $\mathrm{N}$, et al. Adipose tissue remodeling in lipedema: adipocyte death and concurrent regeneration. J Cutan Pathol. 2009; 36(12): 1293-1298, doi: 10.1 I I //j.16000560.2009.01256.x, indexed in Pubmed: 1928I484.

19. Child AH, Gordon KD, Sharpe P, et al. Lipedema: an inherited condition. Am J Med Genet A. 2010; I52A(4): 970-976, doi: 10.1002/ajmg.a.33313, indexed in Pubmed: 203586 II.

20. Bano G, Mansour S, Brice G, et al. Pit-I mutation and lipoedema in a family. Exp Clin Endocrinol Diabetes. 2010; 1 18(6): 377-380, doi: 10.1055/s-0029-1224I54, indexed in Pubmed: 19609847.

21. Warren Peled A, Kappos EA. Lipedema: diagnostic and management challenges. Int J Womens Health. 2016; 8: 389-395, doi: 10.2 I47/IJWH.SI06227, indexed in Pubmed: 27570465. 
22. Stutz JJ, Krahl D. Water jet-assisted liposuction for patients with lipoedema: histologic and immunohistologic analysis of the aspirates of 30 lipoedema patients. Aesthetic Plast Surg. 2009; 33(2): 153-162, doi: 10.1007/s00266-008-92/4-y, indexed in Pubmed: 18663515.

23. Vignes S. [Lipedema: a misdiagnosed entity]. J Mal Vasc. 20I2; 37(4): 213-218, doi: 10.1016/j.jmv.2012.05.002, indexed in Pubmed: 22698628.

24. Schmeller W, Meier-Vollrath I. Lipödem. Gefässchirurgie. 2009; 14(6): 516-522, doi: 10.1007/s00772-009-0747-4.

25. Meier-Vollrath I, Schmeller W. Lipoedema - current status, new perspectives. J Dtsch Dermatol Ges. 2004; 2(3): I8I-186.

26. Fife C, Carter M. Lymphoedema in bariatric patients. J Lymphoedema. 2009; 4(2): 29.

27. Naouri M, Samimi M, Atlan M, et al. High-resolution cutaneous ultrasonography to differentiate lipoedema from lymphoedema. Br J Dermatol. 2010; 163(2): 296-30I, doi: 10.1 III/j.13652133.2010.09810.x, indexed in Pubmed: 20408836.

28. Monnin-Delhom ED, Gallix BP, Achard C, et al. High resolution unenhanced computed tomography in patients with swollen legs. Lymphology. 2002; 35(3): $121-128$, indexed in Pubmed: 12363222.

29. Dimakakos PB, Stefanopoulos T, Antoniades P, et al. MRI and ultrasonographic findings in the investigation of lymphedema and lipedema. Int Surg. 1997; 82(4): 4II-4I6, indexed in Pubmed: $94 \mid 2843$.

30. Duewell S, Hagspiel KD, Zuber J, et al. Swollen lower extremity: role of MR imaging. Radiology. 1992; 184(I): 227-231, doi: 10.1148/radiology.184.1.1609085, indexed in Pubmed: 1609085.

31. Lohrmann C, Foeldi E, Langer M. MR imaging of the lymphatic system in patients with lipedema and lipo-lymphedema. Microvasc Res. 2009; 77(3): 335-339, doi: 10.1016/j.mvr.2009.01.005, indexed in Pubmed: 19323976.

32. Harwood CA, Bull RH, Evans J, et al. Lymphatic and venous function in lipoedema. Br J Dermatol. 1996; 134(I): I-6, indexed in Pubmed: 8745878.

33. Van Geest AJ, Esten, Cambier J, et al. Lymphatic disturbances in lipoedema. Phlebologie. 2003; 32(6): |38-142.

34. Boursier V, Pecking A, Vignes S. Comparative analysis of lymphoscintigraphy between lipedema and lower limb lymphedema. J Mal Vasc. 2004; 29(5): 257-26I, indexed in Pubmed: 15738837.

35. Bilancini S, Lucchi M, Tucci S, et al. Functional lymphatic alterations in patients suffering from lipedema. Angiology. 1995; 46(4): 333-339, doi: 10.1177/000331979504600408, indexed in Pubmed: 7726454.

36. Rasmussen JC, Herbst KL, Aldrich MB, et al. An abnormal lymphatic phenotype is associated with subcutaneous adipose tissue deposits in Dercum's disease. Obesity (Silver Spring). 2014; 22(10): 2186-2192, doi: 10.1002/oby.20836, indexed in Pubmed: 25044620.

37. Beesley V, Janda M, Eakin E, et al. Lymphedema after gynecological cancer treatment : prevalence, correlates, and supportive care needs. Cancer. 2007; 109(12): 2607-26I4, doi: 10.1002/ cncr.22684, indexed in Pubmed: $17474 \mid 28$.

38. Stemmer R. Stemmer's sign - possibilities and limits of clinical diagnosis of lymphedema. Wien Med Wochenschr. 1999; 149 (24): 85-86, indexed in Pubmed: 10378332.
39. Foldi E. The Stemmer sign | Uber das Stemmersche Zeichen. Vasomed. 1997; 9(3).

40. Warren AG, Janz BA, Borud LJ, et al. Evaluation and management of the fat leg syndrome. Plast Reconstr Surg. 2007; I 19(I): 9e-915e, doi: 10.1097/0I.prs.0000244909.82805.dc, indexed in Pubmed: 17255648.

4I. Drozdz K, Chachaj A, Szuba A. The decongestive lymphatic therapy in the massive primary lower limb lymphedema treatment. Acta Angiologica. 2017; 23(3): 130-134, doi: 10.5603/aa.2017.001 I.

42. Ochatek K. The role of compression therapy in venous-lymphatic disturbances. Acta Angiol. 2012; 18(4): 168-76.

43. Herpertz $U$. Range of lipedema at a special clinic for lymphological diseases: Manifestations, combinations and treatment possibilities | Krankheitsspektrum des Lipodems an einer Lymphologischen Fachklinik - Erscheinungsformen, Mischbilder und Behandlungsmoglichkeiten. Vasomed. 1997; 9(5).

44. Müssig K, Gallwitz B. Lipohypertrophy. Dtsch Med Wochenschr. 2006; I3I(33): 1807-1808, doi: 10.1055/s-2006-949|58, indexed in Pubmed: 16902904.

45. Reich-Schupke S, Altmeyer P, Stücker M. Thick legs - not always lipedema. J Dtsch Dermatol Ges. 2013; II(3): 225-233, doi: 10.1 I I I/ddg. 12024, indexed in Pubmed: 23231593.

46. Yeh NC, Yang CY, Chou CW, et al. Madelung's disease. J Clin Endocrinol Metab. 2012; 97(9): 3012-3013, doi: 10.1210/jc.20121649, indexed in Pubmed: 22701018.

47. González-García R, Rodríguez-Campo FJ, Sastre-Pérez J, et al. Benign symmetric lipomatosis (Madelung's disease): case reports and current management. Aesthetic Plast Surg. 2004; 28(2): 108-112.

48. S. B, M. W, A. L, Y. S. Adiposis dolorosa (Dercum's disease): 10-year follow-up. Ann Plastic Surg. 1994; 33: 664-668 .

49. Herbst K, Asare-Bediako S. Adiposis dolorosa is more than painful fat. The Endocrinologist. 2007; 17(6): 326-334, doi: 10.1097/ ten.0b013e318I5942294.

50. Amine B, Leguilchard F, Benhamou C. Dercum's disease (adiposis dolorosa): a new case-report. Joint Bone Spine. 2004; 7 I (2): |47-149, doi: 10.1016/s |297-319x(03)00।39-8.

5I. Beltran K, Herbst KL. Differentiating lipedema and Dercum's disease. Int J Obes (Lond). 2017; 4I (2): 240-245, doi: 10. 1038/ ijo.2016.205, indexed in Pubmed: 27857। 36.

52. Campen R, Mankin H, Louis DN, et al. Familial occurrence of adiposis dolorosa. J Am Acad Dermatol. 200 I; 44(I): 132-136, doi: 10.1067/mjd.200 I. I 10872, indexed in Pubmed: I I 14849 I.

53. Hansson E, Svensson H, Brorson H. Review of Dercum's disease and proposal of diagnostic criteria, diagnostic methods, classification and management. Orphanet J Rare Dis. 2012; 7: 23, doi: 10.1 I86/1750-1 I72-7-23, indexed in Pubmed: 22546240.

54. Garg A. Clinical review: lipodystrophies: genetic and acquired body fat disorders. J Clin Endocrinol Metab. 2011; 96(II): 3313-3325, doi: 10.1210/jc.2011-1159, indexed in Pubmed: 21865368.

55. Kurugöl Z, Ulger Z, Berk O, et al. Acquired partial lipodystrophy associated with varicella. Turk J Pediatr. 2009; 5I(6): 617-620, indexed in Pubmed: 20196400.

56. Goodliffe JM, Ormerod JOM, Beale A, et al. An under-diagnosed cause of leg swelling. BMJ Case Rep. 2013; 2013, doi: 10.1136/ bcr-2013-009538, indexed in Pubmed: 23709549.

57. Fetzer A, Wise $C$. Living with lipoedema: reviewing different self-management techniques. $\mathrm{Br} J$ Community Nurs. 2015; Sup- 
pl Chronic: SI4, SI6-SI4, SI9, doi: 10.12968/bjcn.20I5.20. Sup I0.SI4, indexed in Pubmed: 26418584.

58. Fetzer A. Specialist approaches to managing lipoedema. $\mathrm{Br}$ J Community Nurs. 2016; Suppl: S30-S35, doi: 10.12968/ bjcn.2016.21.Sup4.S30, indexed in Pubmed: 27046426.

59. Wagner S. Lymphedema and lipedema - an overview of conservative treatment. Vasa. 20I I; 40(4): 27I-279, doi: 10.1024/030I1526/a000 I I5, indexed in Pubmed: 21780050.

60. Szolnoky G, Nagy N, Kovács RK, et al. Complex decongestive physiotherapy decreases capillary fragility in lipedema. Lymphology. 2008; 4 I(4): I6I-I66.

6I. Szolnoky G, Varga E, Varga M, et al. Lymphedema treatment decreases pain intensity in lipedema. Lymphology . 201 I; 44(4): 178-182.

62. Tan IC, Maus EA, Rasmussen JC, et al. Assessment of lymphatic contractile function after manual lymphatic drainage using near-infrared fluorescence imaging. Arch Phys Med Rehabil.
20II; 92(5): 756-764.el, doi: 10.1016/j.apmr.2010.12.027, indexed in Pubmed: 21530723.

63. Wienert V, Gerlach H, Gallenkemper G, et al. Leitlinie Medizinischer Kompressionsstrumpf (MKS). Phlebologie. 2018; 35(06): 315-320, doi: 10.1055/s-0038-16390I7.

64. Rapprich S, Dingler A, Podda M. Liposuction is an effective treatment for lipedema-results of a study with 25 patients. J Dtsch Dermatol Ges. 201 I; 9(I): 33-40, doi: 10.1111/j.16100387.2010.07504.x, indexed in Pubmed: 21166777.

65. Schmeller W, Hueppe M, Meier-Vollrath I. Tumescent liposuction in lipoedema yields good long-term results. Br J Dermatol. 2012; 166(I): 161-168, doi: 10.1111//j.1365-2133.2011.10566.x, indexed in Pubmed: 21824127.

66. Peled AW, Slavin SA, Brorson H. Long-term Outcome After Surgical Treatment of Lipedema. Ann Plast Surg. 2012; 68(3): 303-307, doi: 10.1097/SAP.0b0I3e3 I82 I579le, indexed in Pubmed: 21629090. 